\title{
New Method of Content Based Image Retrieval based on 2-D ESPRIT Method and the Gabor Filters
}

\author{
Chawki Youness*, El Asnaoui Khalid, Ouanan Mohammed, Aksasse Brahim \\ ASIA Team, M2I Laboratory, Department of Computer Sciences, \\ Faculty of Science and Techniques, Moulay Ismail University, Morocco \\ ${ }^{*}$ Corresponding author, email: youness.chawki@gmail.com
}

\begin{abstract}
We propose, in this paper, a new method for Content Based Image Retrieval (CBIR) by exploiting the digital image content. Our method is based on the representation of the digital image content by a characteristics vector of the indexed image. Indeed, we have exploited the image texture to extract its characteristics and for constructing a new descriptor vector by combining the Bidimensional High Resolution Spectral Analysis 2-D ESPRIT (Estimation of Signal Parameters via Rotationnal Invariance Techniques) method and Gabor filter. To evaluate the performance, we have tested our approach on Brodatz image database. The results show that the representation of the digital image content appears significant in research of imaging information.
\end{abstract}

Keywords: indexing, search images by content, high resolution, spectral analysis, 2-d esprit, Gabor filter

Copyright $@ 2015$ Institute of Advanced Engineering and Science. All rights reserved.

\section{Introduction}

The techniques for indexing and information retrieval have been developed since the 70s. First, Text-Based Image Retrieval (TBIR) methods were the first methods used. However, these methods have major drawbacks: the first is related to the amount of work involved in the process, and the second is the lack of accuracy in the description in large databases. Therefore, to overcome this problem, it is important to develop others systems using inherent information of the content. Hence Content-Based Image Retrieval (CBIR) System based on the images digital content is developed. Indeed, these approaches consist in representing every image by a set of visual features such as the color, the shape and the texture. These visual characteristics are then used to compare and to find a query image in a database.

Texture is the second visual attribute widely used in image search by content. It allows filling the gaps that the color is unable to do, especially when the color distributions are very close. More specifically, the texture can be viewed as a set of pixels (grayscale) spatially arranged in a number of spatial relationships, and creating a homogeneous region. Thus, several approaches and models $[1,2]$ have been proposed for modeling the texture, which we quote: statistical approaches, geometric approaches and the frequency approaches. For the latter, the Gabor filters are the most known and most used method.

Gabor filters are introduced by Hungarian Physicist Dennis Gabor in 1946 [3]. They prove to be an interesting tool for texture analysis and are widely adopted in the literature. The advantage of these filters is that their functioning is close to the human visual treatments, and they have the advantage of being programmable in frequency and in orientation. Indeed, Gabor filters find their place in several areas such as: segmentation [4], pattern recognition [5, 6], classification [7, 8], content based image retrieval [9, 10]. The texture parameters are determined by calculating the average and the variance of the image filtered by Gabor filter.

The aim for spectral analysis is to characterize the frequency content of images or signals in general. We often use, for images, the frequency information to extract texture content. The high resolution spectral methods called parametric method can be divided into two categories: the first contains pseudo-spectrum methods while the second family groups the subspace methods and are based on the exploitation of the inherent structure of the model. The 2-D ESPRIT method [11-13] is the most famous and the used method in bidimensional frequency estimation. It provides the pair frequency and its corresponding orientation 
automatically with good precision. These last parameters will be injected into the Gabor filter. Since it is depending on theme.

This work presents a new approach for indexing and content based image retrieval. The following sections are arranged as follows: Section 2 describes the 2-D ESPRIT method, and then the Gabor filters are presented in Section 3. Section 4 explains our new approach. Subsequently the experimental results are presented in Section 5. Finally we conclude our work with conclusions and perspectives.

\section{The 2-D ESPRIT Method [12]}

The frequency extracted by the 2-D ESPRIT method is in the form of two components which are a frequency couple: one component is on the first frequency axis and the other component is on the second frequency axis; therefore the frequency module is defined as follow:

$$
F_{i}=\sqrt{f_{1 i}^{2}+f_{2 i}^{2}}
$$

The corresponding rotation angle $\theta_{i}$ is giving by:

$$
\theta_{i}=\arctan \left(\frac{f_{2 i}}{f_{1 i}}\right)
$$

Figure 1 depicts the relationship between the frequency components $f_{1 i}, f_{2 i}$, and the module $F_{i}$, and rotation angle $\theta_{i}$ :

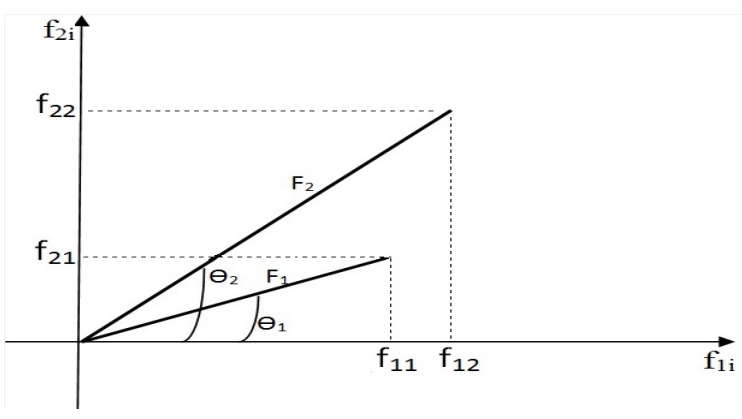

Figure 1. Relationship between the two components and the rotation angle

Thus ESPRIT 2-D provides us the frequencies and its orientations that will be the input parameters of Gabor filters.

In our case, the number of frequencies $F_{i}$ and orientations $\theta_{i}$ are the same as number of the frequencies components.

\section{The Gabor Filters}

Gabor filters are multi-channel filtering techniques that allow the description of textures localized in frequency and orientation. In other words, the characteristic calculations are operated on each of the pixels. These contain the intensity variations on a smaller scale of frequency and orientation. The gabor function can be written as follows:

$$
G(x, y, \theta, f)=\exp \left(-\frac{1}{2}\left(\frac{x_{\theta}^{2}}{\sigma_{x}^{2}}+\frac{y_{\theta}^{2}}{\sigma_{y}^{2}}\right)\right) \cos \left(2 \pi f x_{\theta}\right)
$$


Where:

$$
\begin{aligned}
& x_{\theta}=x \cos \theta+y \sin \theta \\
& y_{\theta}=-x \sin \theta+y \cos \theta
\end{aligned}
$$

$\theta$ is the orientation, $F$ the frequency and $\sigma_{x}$ (respectively $\sigma_{y}$ ) the standard deviation of the gaussian according the $x$ axis ( $y$ axis respectively). By applying this function as a convolution mask, we define a convolution filter called Gabor filter.

For a given frequency $F$ and a given orientation $\theta$, we have a Gabor filter, allowing towards the end to have several filters by changing the orientation and also the frequency, and finally to have a set of filters called banc of filters.

After constructing the Gabor filters, they are applied to a $M x N$ image in order to extract the average and the variance of filtered image by each Gabor. The average and variance have the following expressions respectively:

$$
\begin{aligned}
\mu_{m, n} & =\frac{\sum_{x} \sum_{y}\left|G_{m, n}(x, y)\right|}{M x N} \\
\sigma_{m, n} & =\sqrt{\frac{\sum_{x} \sum_{y}\left(\mid G_{m, n}(x, y)-\mu_{m, n}\right)^{2}}{M x N}}
\end{aligned}
$$

The values of $\sigma_{m, n}$ and $\mu_{m, n}$ will represent the components of our descriptor vector.

\section{The Proposed Approach}

Here, we propose a new method for indexing and content based image retrieval using the bidimensional high resolution spectral analysis methods especially the 2-D ESPRIT method and Gabor filters. Indeed, each image is characterized by the pair given by the Gabor filters for each orientation and each frequency, this last parameters are given by the 2-D ESPRIT method applied on the original image.

Indexing and content based image retrieval system includes two main phases, the indexing and the search phase, Figure 2 shows these two phases.

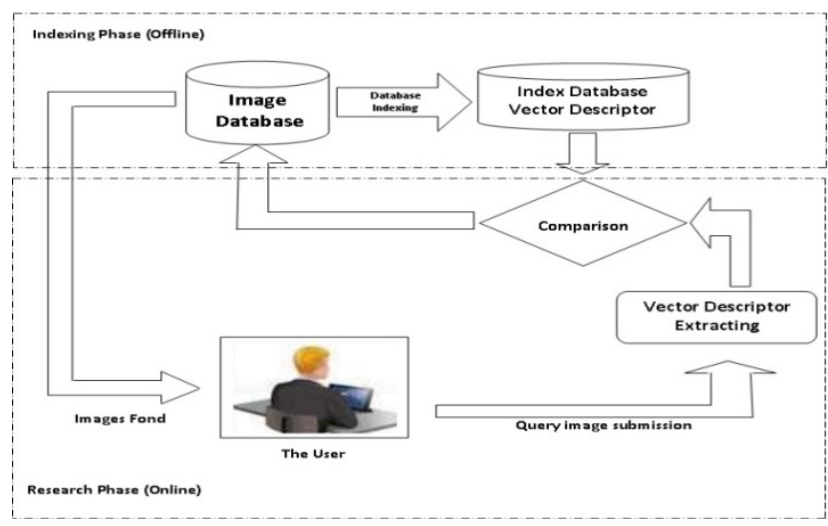

Figure 2. Overall architecture of content based image retrieval

The first involves extracting the characteristics of each image and they are stored in a database. This step is running in Offline. 
The second phase is running in Online, the system takes a query image, extract its digital characteristics, and then measure the distance between the query image vector descriptor and those in the database.

The main steps in features extraction of textures image using the 2-D ESPRIT method and Gabor filters are illustrated and summarized in Figure 3.

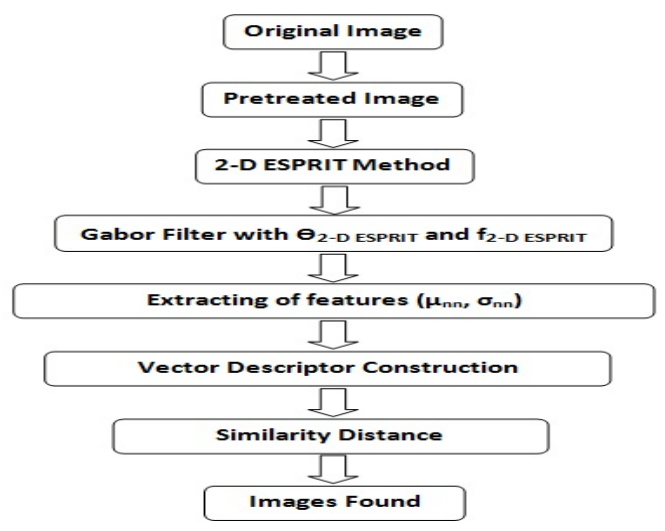

Figure 3. The Main steps of the proposed approached are cited bellow

1) Frequency pair and orientation extraction: The first step is to extract from the original image by using the 2-D ESPRIT method the frequency pairs $\left(f_{1 i}, f_{2 i}\right)$. Furthermore, we calculate the frequency module and the orientation. These parameters will be injected into Gabor filters.

2) Average calculation: After applying the Gabor filters on the image, with different orientations $\theta_{m}$ and frequency $f_{m}$, we calculate the average $\mu_{m}$ that characterizes the luminous intensity of the image corresponding to the average grayscale of all pixels in the image (Equation 5).

3) Standard deviation calculation: The standard deviation $\sigma_{m}$ characterizes the variation of the average intensity of all pixels. It corresponds to the change of the image contrast (Equation 6).

After extracting the characteristics of each image, we construct the vector descriptor in which each component represents two values: the average and the variance. In other words, for each frequency and each orientation, we have a pair $(\mu, \sigma)$. Finally, the descriptor vector will be defined as follows:

$$
V=\left(\mu_{0}, \sigma_{0}, \mu_{1}, \sigma_{1}, \ldots ., \mu_{m}, \sigma_{m}\right)
$$

The algorithm of our approach is the following:

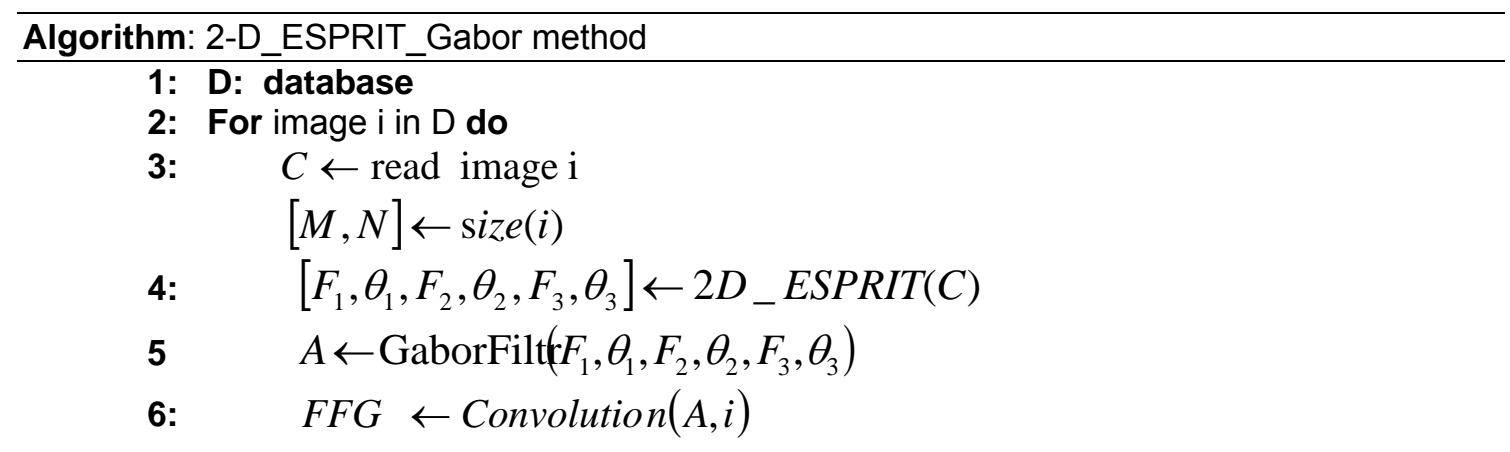




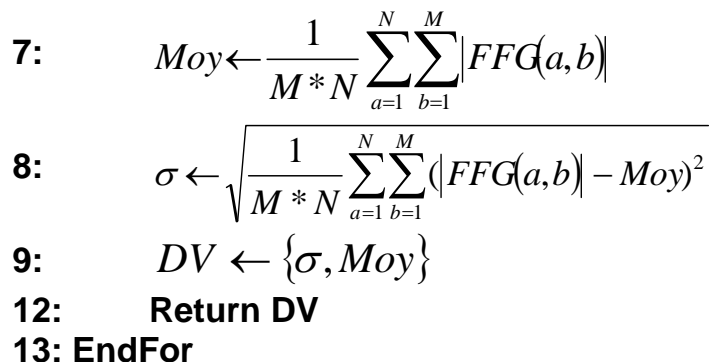

The similarity measure between a query image $Q$ and a target image $T$ is carried out by calculating, for each value of the vector, of the Swain distance [14] $D(Q, T)$ defined by:

$$
D(Q, T)=\sum \sum d_{m}(Q, T)
$$

Where,

$$
d_{m}=\left|\left(\mu_{m}^{Q}-\mu_{m}^{T}\right),\left(\sigma_{m}^{Q}-\sigma_{m}^{T}\right)\right|
$$

\section{Experemental Results}

In our experiment, we perform detailed performance comparison to evaluate the efficiency of the proposed approach. Indeed, we have tested our approach on the texture Brodatz database [15] including 13 texture classes, 16 samples each. All images are in grayscale BMP format, each 256 by 256 pixels. Figure 4 shows examples of images from Brodatz database.
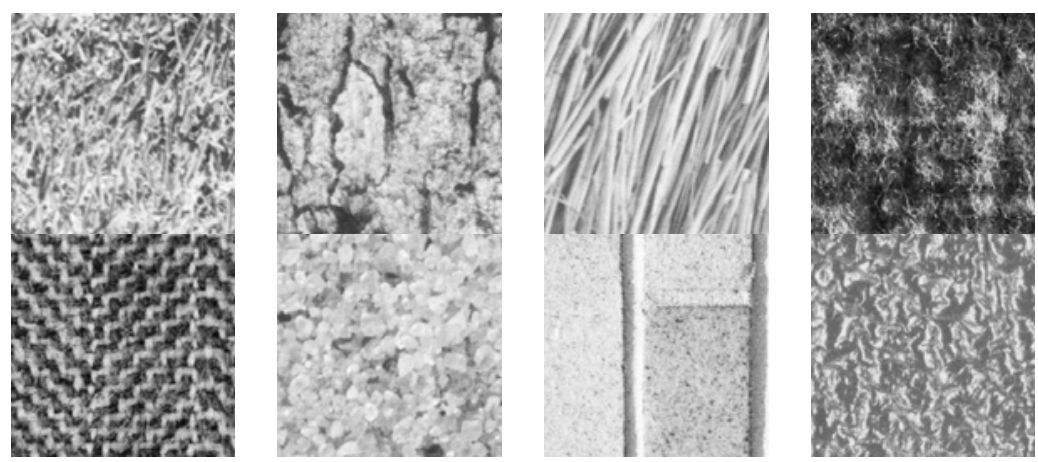

Figure 4. Examples of images from Brodatz database

\subsection{Implementation}

In the implementation phase, we have used a computer, Processor: Intel(R) Core (TM) 2 CPU T5870 @ 2,00 GHz, 2,00 GHz, 2Go RAM, Windows 7.

We have developed an user graphic interface for displaying the results. We just display the 14 images similar to the query image.

Figure 5 shows an example of query image (D16_001 image), our approach has returned 14 relevant similar images while figure 6 illustrates an another example of query image (D84_001) with 12 images similar images including two not relevant images (D94_001 and D38_010). 
Table1. The precision end the recall for each class on Brodatz images database

\begin{tabular}{cccc}
\hline Classes & Found images & Precison (\%) & Recall (\%) \\
\hline D9 & 169 & 68,75 & 60,16 \\
D12 & 163 & 71,43 & 62,50 \\
D15 & 98 & 70,09 & 61,89 \\
D16 & 55 & 77,68 & 87,97 \\
D19 & 15 & 84,38 & 65,23 \\
D24 & 127 & 78,57 & 68,75 \\
D29 & 174 & 89,29 & 78,13 \\
D38 & 163 & 77,23 & 67,58 \\
D68 & 38 & 100,00 & 87,50 \\
D84 & 167 & 77,68 & 64,09 \\
D92 & 178 & 82,14 & 71,88 \\
D94 & 165 & 81,25 & 71,09 \\
D112 & 167 & 83,93 & 73,44 \\
& & Average Precision (\%) & Average Recall (\%) \\
\hline
\end{tabular}

The Table 1 shows the precision and recall of each class of Brodatz database. We can see that the precision is more than $70 \%$ for all classes and the average precision is equal to $80,19 \%$, which shows that our approach is accurate.

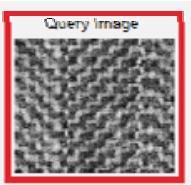

146, 12 omp

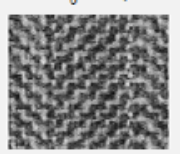

016 , 02 bmp

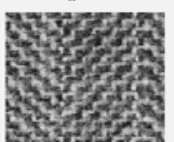

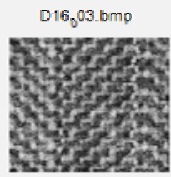

U16, $13 . \mathrm{omp}$

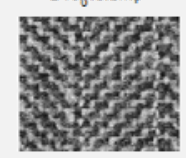

$016_{\mathrm{v}} 14 \mathrm{bmp}$

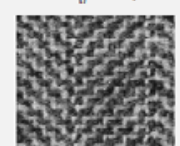

Figure 5. Similar images for D16_001 class

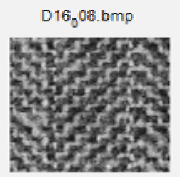

U16 $09 \mathrm{gmp}$

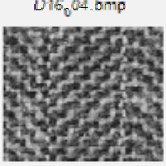

$016_{0} 16 \mathrm{bmp}$
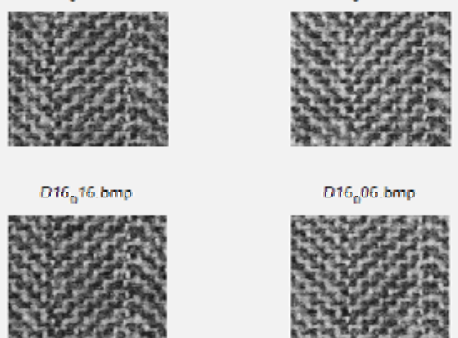

$6.05 .8 \mathrm{mp}$

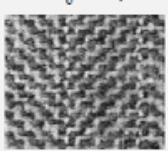

$016_{10} 01 \mathrm{bmp}$
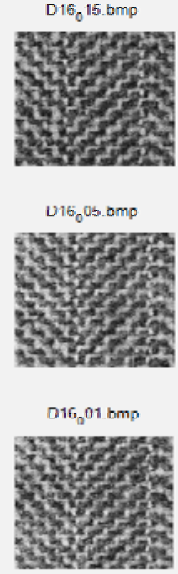

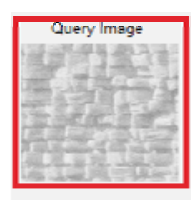

nas $0.3 \mathrm{hmp}$

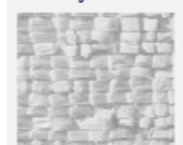

บะ4. 0 0u. bmp

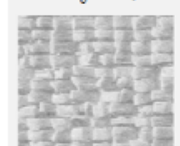

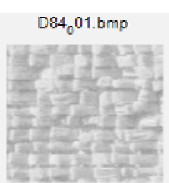

no4 ${ }_{0} 12$ hrmp

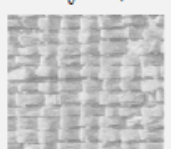

U84, 06 . bmp

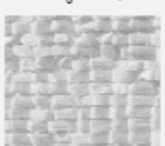

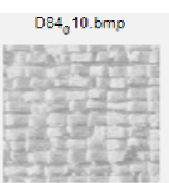

ne4, $11 \mathrm{bmg}$

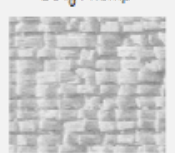

V94, 01 bmp

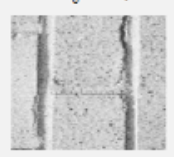

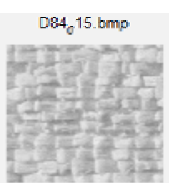
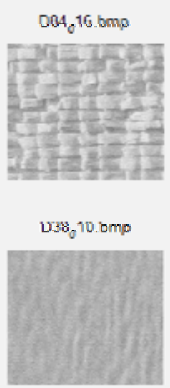

nRA $07 \mathrm{hmm}$

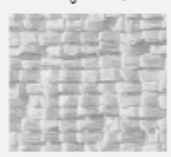

naA, ori bann

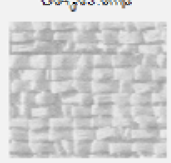

vo4, 09 bmp

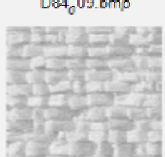

Figure 6. Similar images for D84_001 class 


\subsection{Evaluation Protocol}

In the information retrieval system, the user is interested to relevant system responses. So the information search systems require the precision evaluation of the response. This type of evaluation is considered as the research performance evaluation. Thus, to evaluate the efficiency of our system, we are interested to calculate the two most commonly used measures. Namely: the precision and the recall, these two parameters measure the relevance of our system.

Recall is the ratio between the number of relevant images in the set of images found and the number of relevant image in the image database.

$$
\text { Recall }=\frac{R_{a}}{R}
$$

Where:

$R_{a}$ : Number of relevant images in the set of responses.

$R:$ Number of relevant images in the image database.

Precision is the ratio between the number of relevant images in the set of images found and the number of relevant image.

$$
\text { Precision }=\frac{R_{a}}{A}
$$

Where:

$A$ : Number of images in the set of responses.

The curve 7 and 8 show the precision average and the recall average respectively of our approach compared with the Gabor method. We see that our method is more accuracy than the Gabor method.

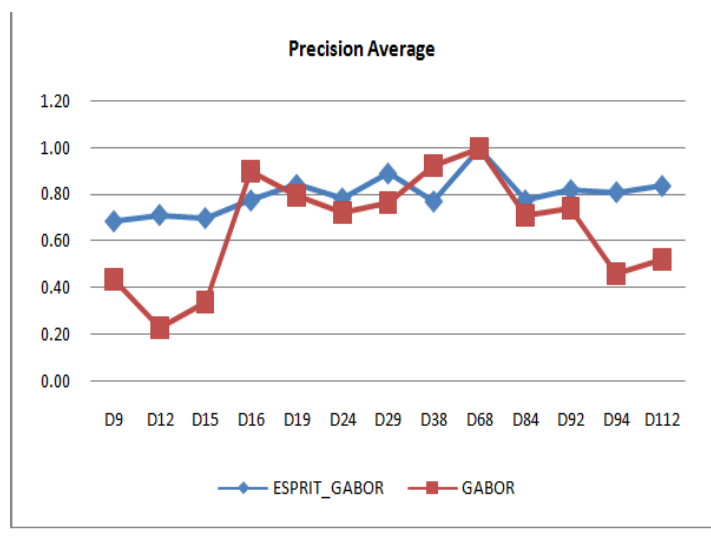

Figure 7. Precision curve of our approach compared with the Gabor Filter

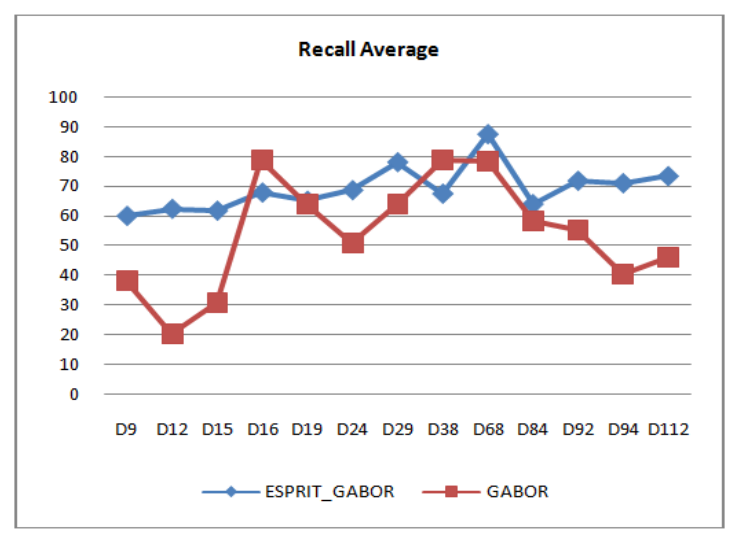

Figure 8. Recall curve of our approach compared with the Gabor Filter method

\section{Conclusion \& Perspectives}

In this work, we have presented a new method for indexing and content based image retrieval using Gabor filters powered or feed by the frequency and orientation given by the bidimensional high resolution spectral analysis especially 2-D ESPRIT. This approach is applied to the Brodatz database containing 208 images and has given good results making it possible to say that our approach is accurate.

In terms of perspectives, we will try to apply our approach initially on image databases other than textured databases, and then we will combine the High Resolution Spectral Analysis methods with other visual characteristics such as the shape and color. 


\section{References}

[1] J Zhang, T Tan. Brief review of invariant texture analysis methods. Pattern Recognition. 2002; 35: 735-747.

[2] D Gabor. Theory of communication. Journal of the Institute of Electrical Engineers. 1946; 93: 429-457.

[3] J Zhang, T Tan, L Ma. Invariant Texture Segmentation Via Circular Gabor Filters. Pattern Recognition, Proceedings 16th International Conference. 2002; 2: 901-904.

[4] CS Avila, RS Reillo. Two different approaches for iris recognition using Gabor filters and multiscale zero-crossing representation. Pattern Recognition. 2005; 38: 231-240.

[5] K Thangairulappan, JBV Jeyasingh. Face Representation Using Combined Method of Gabor Filters, Wavelet Transformation and DCV and Recognition Using RBF. Journal of Intelligent Learning Systems and Applications. 2012; 4: 266-273.

[6] S Arivazhagan, L Ganesan, SP Priyal. Texture classification using Gabor wavelets based rotation invariant features. Pattern Recognition Letters. 2006; 27: 1976-1982.

[7] Rahmadwati, G Naghdy, M Ros, C Todd, E Norahmawati. Cervical Cancer Classification Using Gabor Filters. Healthcare Informatics, Imaging and Systems Biology (HISB), First IEEE International Conference. 2011: 48- 52.

[8] N Bourkache, S Sidhom, M Laghrouche. Apprentissage numérique pour la recherche d'informations en imagerie médicale: Modélisation des filtres de Gabor. International symposium ISKO-Maghreb' 13, Concepts and Tools for Knowledge Management (KM). Marrakech, Morocco. 2013.

[9] M Agarwal, RP Maheshwari. Content Based Image Retrieval Based on Log Gabor Wavelet Transform. Advanced Materials Research, Trans Tech Publications. 2012: 871-878.

[10] Y Hua. Estimating Two-Dimensional Frequencies by Matrix Enhancement and Matrix Pencel. IEEE Transactions on Signal Processing. 1997; 40(9).

[11] $P$ Vanpoucke, M Moonen, $Y$ Berthoumieu. An efficient subspace algorithm for $2 D$ harmonic retrieval. IEEE International Conference on Acoustics, Speech and Signal Processing. 1994: 461-464.

[12] Y Zhou, DZ Feng, JQ Liu. A novel algorithm for two-dimensional frequency estimation. Signal Processing. 2007; 87: 1-12.

[13] S Rouquette, M Najim. Frequencies and damping factors by two-dimensional ESPRIT type methods. IEEE Transactions on Signal Processing. 2001; 49(1): 237-245.

[14] Y Berthoumieu, M El Ansari, B Aksasse, M Donias, M Najim. A 2-D Robust high resolution frequency estimation approach. Signal Processing. 2005; 85(6): 1165-1188.

[15] USC-SIPI image database. Available online at: http://sipi.usc.edu/database/. 1977 